\title{
ARTICLE
}

Received 27 Jun 2013 | Accepted 24 Nov 2013 | Published 9 Jan 2014 | Updated 20 Feb 2014

DOI: $10.1038 /$ ncomms 4015

\section{Manipulating surface reactions in lithium-sulphur batteries using hybrid anode structures}

Cheng Huang ${ }^{1}$, Jie Xiao ${ }^{1}$, Yuyan Shao', Jianming Zheng ${ }^{1}$, Wendy D. Bennett ${ }^{1}$, Dongping Lu', Laxmikant V. Saraf', Mark Engelhard', Liwen Ji ${ }^{1}$, Jiguang Zhang ${ }^{1}$, Xiaolin Li ${ }^{1}$, Gordon L. Graff ${ }^{1}$ \& Jun Liu ${ }^{1}$

Lithium-sulphur batteries have high theoretical energy density and potentially low cost, but significant challenges such as severe capacity degradation prevent its widespread adoption. Here we report a new design of lithium-sulphur battery using electrically connected graphite and lithium metal as a hybrid anode to control undesirable surface reactions on lithium. Lithiated graphite placed in front of the lithium metal functions as an artificial, self-regulated solid electrolyte interface layer to actively control the electrochemical reactions and minimize the deleterious side reactions, leading to significant performance improvements. Lithiumsulphur cells incorporating this hybrid anodes deliver capacities of $>800 \mathrm{mAhg}^{-1}$ for 400 cycles at a high rate of $1,737 \mathrm{~mA} \mathrm{~g}^{-1}$, with only $11 \%$ capacity fade and a Coulombic efficiency $>99 \%$. This simple hybrid concept may also provide scientific strategies for protecting metal anodes in other energy-storage devices.

\footnotetext{
${ }_{1}^{1}$ Pacific Northwest National Laboratory, Richland, Washington 99352, USA. Correspondence and requests for materials should be addressed to J.L. (email: Jun.Liu@pnnl.gov).
} 
A lthough rechargeable lithium (Li) ion batteries (LIBs) are widely studied for application in portable electronic devices and vehicle electrification, they cannot store sufficient energy for the extended driving range required by electric vehicles ${ }^{1-7}$. Alternative energy-storage systems with much higher theoretical specific energy are needed ${ }^{8-11}$. Among these technologies, Li-sulphur (Li-S) batteries hold great promise ${ }^{8-19}$. Theoretically, a Li-S battery has a specific capacity and energy of $1,675 \mathrm{mAh} \mathrm{g}^{-1}$ and $2,600 \mathrm{Wh} \mathrm{kg}^{-1}$, respectively, much higher than those of traditional LIBs. Sulphur is also of low cost, and is abundant and non-toxic, making this system attractive for largescale applications. However, many obstacles in this system still need to be overcome for practical applications. The major issue associated with Li-S cells is the formation of soluble long-chain polysulphides during discharge/charge. The gradual loss of active mass from the cathode into the electrolyte and onto the Li metal anode leads to 'shuttle reactions', severe self-discharge, low efficiency and fast capacity decay on cycling, which are commonly observed in $\mathrm{Li}-\mathrm{S}$ batteries.

Various strategies have been employed to address the polysulphide shuttle challenges in Li-S cells. For cathodes, nanostructured hosts such as mesoporous carbon, graphene, carbon nanofibres ${ }^{16,20-26}$, oxygenated porous architectures ${ }^{27}$ or conductive polymers have been used to trap sulphur ${ }^{20,28-31}$. One example is Nazar's precise constraint of sulphur within a mesoporous carbon framework ${ }^{16}$. The use of an ordered mesoporous carbon composite provides both an electronic percolation path and an adequately controlled porosity that confines a significant portion of the electrochemically generated polysuphide species in the cathode. Other approaches focus on the electrolyte, including the selection of appropriate solvents $^{32,33}$, dual electrolytes ${ }^{34}$, electrolyte additives ${ }^{13,35-39}$ or solid-state electrolytes ${ }^{40}$. Scrosati and co-workers ${ }^{17,41,42}$ used a gel-type polymer electrolyte membrane as a physical barrier to block the dissolved sulphide species within the cathode compartment and lessen the deleterious side reactions at the anode. Investigation of new binders ${ }^{43}$ has also been reported in an attempt to improve the integrity of the electrode structure, facilitate electron transport and improve $\mathrm{Li}^{+}$ion diffusion $^{21}$.

In addition to the sulphur dissolution problem at the cathode, other studies suggest that continuous contamination of polysulphide species on the Li metal anode is equally, if not more severely, damaging to long-term cell performance, but this problem has received much less attention. For any rechargeable batteries employing $\mathrm{Li}$ metal as the anode, two major failure mechanisms are typically associated with the system. One is the uncontrolled dendrite formation ${ }^{44}$, whereas the other is the continuous evolution of a porous, or mossy, Li metal structure that lowers cell efficiency ${ }^{45}$. The former presents serious safety issues, particularly at high charging rates, whereas the latter continually erodes the anode. This chemical and electrochemical corrosion repeatedly exposes new $\mathrm{Li}$ surface area that further reacts with the electrolyte to form unstable solid-state electrolyte interface (SEI) layers, thus increasing internal cell resistance and accelerating capacity fade ${ }^{22-25}$. For Li-S batteries, where Li metal is coupled with sulphur cathode, the deposition/reduction of insoluble short-chain polysulphides on a $\mathrm{Li}$ anode partially mitigate the dendrite growth problem ${ }^{33,46}$. However, the problem of continuous Li erosion still exists and is compounded with the presence of dissolved polysulphides that also get involved in the continuous SEI formation ${ }^{47}$. Sion Power found that $\mathrm{LiNO}_{3}$, used as an electrolyte additive, helps minimize complicated deleterious reactions between polysulphides and $\mathrm{Li}$, resulting in improved cell life $^{13}$. Tarascon and colleagues ${ }^{48}$ also reported that direct coating of the $\mathrm{Li}$ anode by sulphur effectively stabilizes the cycling of Li-S batteries. Other work also suggests that even a $\mathrm{Li} / \mathrm{Li}_{2} \mathrm{~S}_{9}$ liquid cell can be cycled if an appropriate protective/passivation film can be formed on the $\mathrm{Li}$ metal anode ${ }^{37,49}$. A recent publication, monitoring the content of dissolved sulphur with cycling, further indicates that factors in addition to the dissolved sulphur may be the primary reason for the fast capacity fade in Li-S cells 50

On the basis of the above considerations, further improvements in Li-S cell performance may be achieved by concentrating on advanced anode designs. We speculate that rather than simply using an electrolyte additive or a physical barrier (film) to protect the metal anode, it may be possible to design a completely different anode structure where the interfacial redox reaction is shifted away from the metal surface. To test this hypothesis, a compacted graphite film is connected with $\mathrm{Li}$ foil as an integrated anode and tested against a sulphur cathode ${ }^{20}$. Figure 1 shows the schematic of proposed hybrid anode placed in a Li-S battery. The graphite/Li connected in parallel forms a shorted cell where the graphite is always lithiated at equilibrium and maintains a pseudo-equal potential with the Li metal. As such, it functions as an artificial SEI layer of $\mathrm{Li}$ metal that supplies $\mathrm{Li}^{+}$ions on demand, while minimizing direct contact between soluble polysulphides and the metal surface.

\section{Results}

Cell configuration and cell performance. In the hybrid anode design, a graphite film is placed between the Li foil and separator, and then electrically connected with the Li metal to form a parallel anode (Fig. 1). Once immersed in the electrolyte, Li ions immediately intercalate into the graphite, which persists in the lithiated state because this hybrid anode is, in fact, a shorted cell.

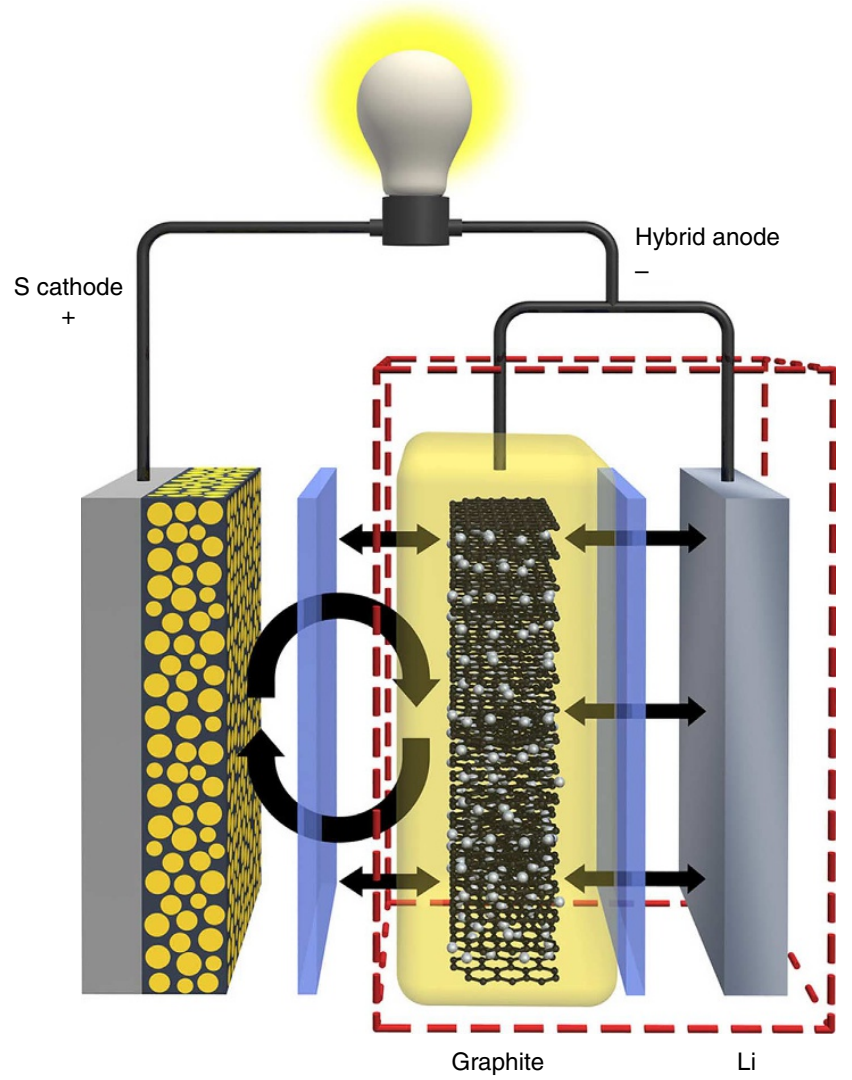

Figure 1 | Schematic of the hybrid anode design. Schematic of the hybrid anode design to manipulate the surface reactions on Li-S batteries. 
a

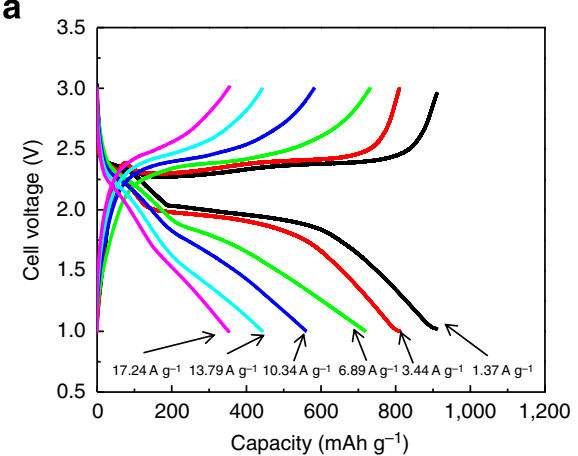

C

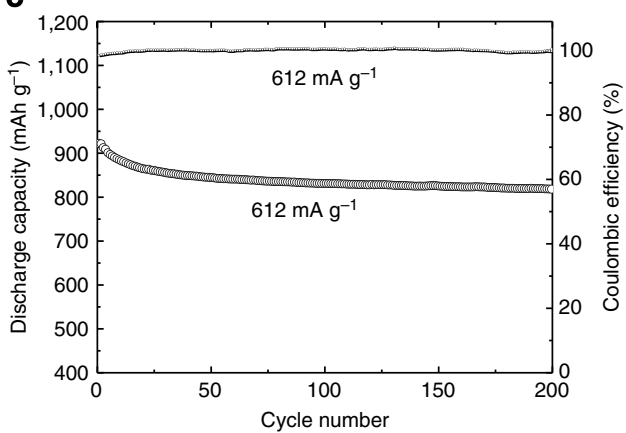

b

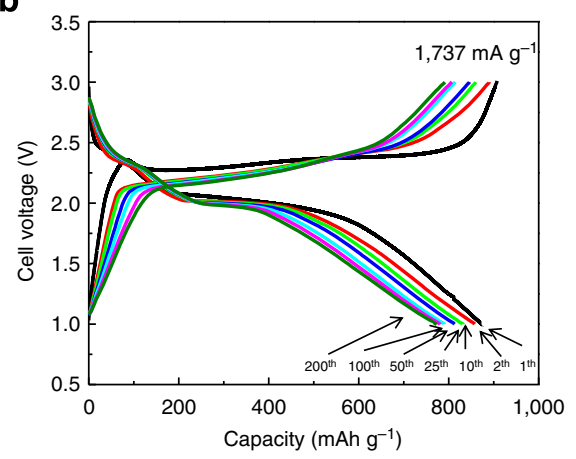

d

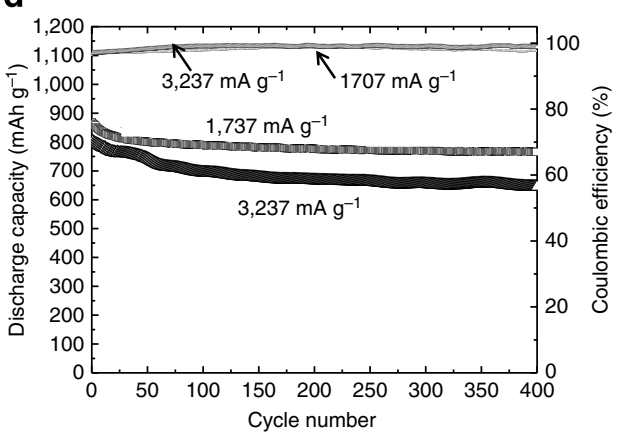

Figure 2 | Electrochemical behaviours of Li-S cells using the proposed hybrid anode. (a) Charge-discharge curves of hybrid Li-S cells at different rates. (b) Cycling ability of the hybrid Li-S cell at a rate of $1,737 \mathrm{mAg}^{-1}$. (c,d) Long-term cycling behaviour and corresponding Coulombic efficiencies of hybrid Li-S cells at different rates.

Using this anode concept, the hybrid $\mathrm{Li}-\mathrm{S}$ cell delivers a reversible capacity of $>900 \mathrm{mAhg}^{-1}$ at $1,370 \mathrm{mAg}^{-1}(\sim 0.8 \mathrm{C})$ as shown in Fig. 2a. Even at a rate of $13,790 \mathrm{~mA} \mathrm{~g}^{-1}(\sim 8 \mathrm{C})$, cell capacity exceeds $450 \mathrm{mAhg}^{-1}$, demonstrating exceptional rate performance. In addition, marked improvement in capacity retention after repeated cycling is observed (Fig. 2b). Figure 2c,d compares the long-term cycling behaviour and Coulombic efficiency of Li-S cells containing hybrid anode at different current density. Significantly improved cycling stability has been observed at all rates. For example, at $612 \mathrm{mAg}^{-1}$, the discharge capacity becomes stable after 50 cycles, maintaining at $\sim 850 \mathrm{mAh} \mathrm{g}^{-1}$ for $>200$ cycles with a small initial capacity loss. The Columbic efficiency is always $>99.5 \%$ over the entire cycling test (Fig. 2c), reflecting that the shuttle mechanism is significantly mitigated in this fundamentally different design of $\mathrm{Li}-\mathrm{S}$ cells. Similar performance enhancements were observed at increased rates and over longer cycling times (Fig. 2b,d), strongly suggesting that this innovative anode concept plays a key role in extending the cycling life of this system. At $1,737 \mathrm{~mA} \mathrm{~g}^{-1}$, the hybrid $\mathrm{Li}-\mathrm{S}$ cell retains a capacity of $>800 \mathrm{mAh} \mathrm{g}^{-1}$ after 400 cycles, corresponding to only $11 \%$ fade and a Coulombic efficiency of $>99 \%$. The electrochemical performances of $\mathrm{Li}-\mathrm{S}$ batteries without hybrid anode design but using the same sulphur cathode are also included in Supplementary Information for comparison (Supplementary Fig. S1).

Materials characterization. The cycled anode was further investigated after extensive cycling (Fig. 3). Energy dispersive spectroscopy, X-ray diffraction and X-ray photoelectron spectroscopy uncover that the chemical composition on $\mathrm{Li}$ metal surface in traditional Li-S cells is complex and comprises sulphide, sulphate, fluoride and carbonate compounds (Supplementary Fig. S2). Unlike the traditional SEI film formed on the anode surface, the chemical composition of surface film on the anode for Li-S batteries is more sophisticated, as ether-based solvents have been used and polysulphides get involved in the SEI growth ${ }^{51}$. Continued reactions between $\mathrm{Li}$ and dissolved polysulphide create an unstable reaction zone that comprises complicated sulphur-containing chemicals, lowering the Coulombic efficiency (Supplementary Fig. S1). Similar SEI composition is expected on graphite surface, as in the same solution their surface chemistry is quite similar to each other ${ }^{52}$. The surface of Li metal harvested from the hybrid electrode remains relatively clean and free of polysulphide deposits. Careful compositional and structural analysis on the cycled hybrid anode suggests that although some polysulphides are deposited on the graphite surface (Fig. $3 c$ and Supplementary Fig. S3), the undesired parasitic reactions and accompanying sulphur contamination is greatly decreased on the Li metal (Fig. 3d-f). In contrast, the $\mathrm{Li}$ anode dissembled from traditional cycled Li-S batteries witnessed a large amount of S-containing species not only on Li metal surface but deeply penetration into bulk Li (Fig. 3g-i).

Graphite loading. At first glance, hybrid anode in its current form (Fig. 1) may affect the energy density of the whole cell, as it incorporates additional accessory weights. However, it was found that the ratio between sulphur and graphite also directly influences the cycling stability (Supplementary Fig. S4). That is, the higher the $\mathrm{S} / \mathrm{LiC}_{6}$ ratio (thinner graphite), the more stable the cycling. In other words, to fully utilize the hybrid concept, the weight of graphite needs to be minimized while the sulphur loading has to be maximized. The weight burden from graphite is therefore largely reduced. Neither gravimetric nor volumetric energy densities of the whole Li-S battery adopting the hybrid anode will be influenced significantly. 
a

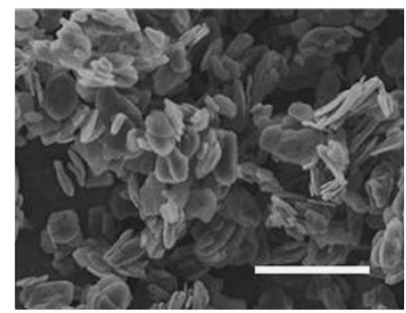

d

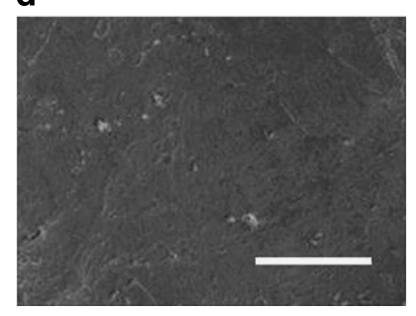

g

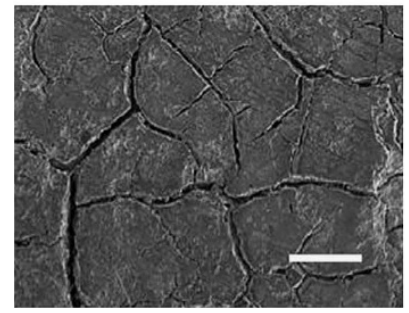

b

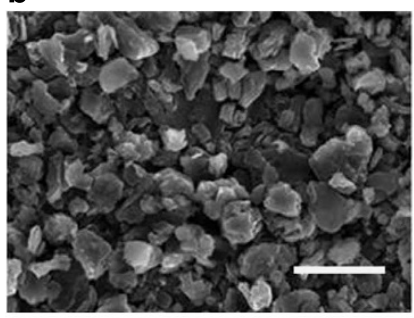

e

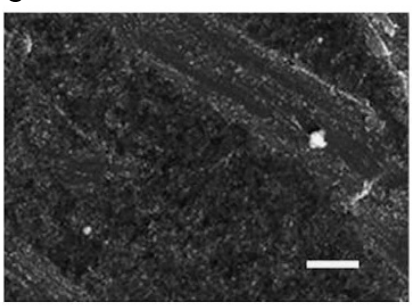

h

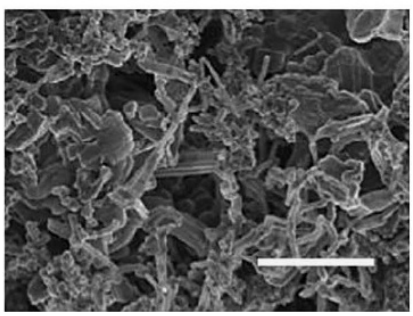

C

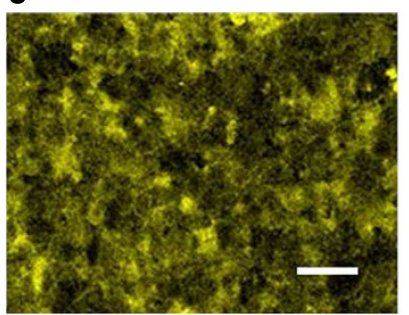

f

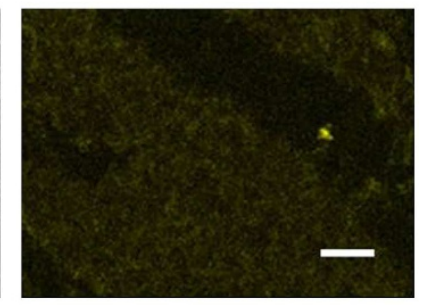

i

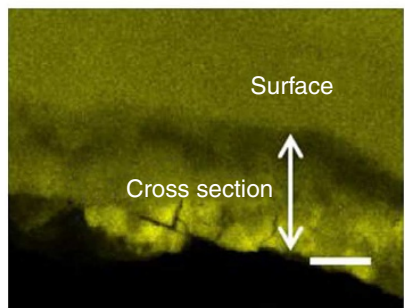

Figure 3 | Microstructure characterization of the hybrid anode. Scanning electrom microscopy (SEM) images of (a) native graphite and (b) graphite

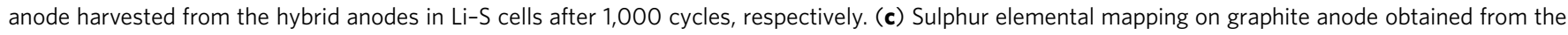
hybrid anodes after 1,000 cycles. (d,e) SEM images of the surface view of Li metal anode from the hybrid anodes after 1,000 cycles at different magnifications. (f) Sulphur elemental mapping of Li metal surface from the hybrid anodes after 1,000 cycles. The current density used for the 1,000 cycles was $3,237 \mathrm{mAg}^{-1}$. ( $\left.\mathbf{g}, \mathbf{h}\right)$ SEM images of the surface view of Li metal anode from traditional Li-S cells after 1,000 cycles at different magnifications. (i) S-elemental mapping of the Li metal anode from traditional Li-S cells. The current density used for the 1,000 cycles was $2,042 \mathrm{~mA} g-1$. Scale bar, $50 \mu \mathrm{m}$ (a-c,g,i), $40 \mu \mathrm{m}$ (d), $10 \mu \mathrm{m}$ (e,f) and $3 \mu \mathrm{m}$ (h).

\section{Discussion}

The exact mechanisms controlling the hybrid electrode are still under investigation. One possible explanation is that the lithiated graphite may function as a physical barrier that decreases the contact area of soluble species in the electrolyte with Li metal. Physical absorption of polysulphide species on graphite may reduce transport of soluble intermediates to the $\mathrm{Li}$ anode. Control cells were assembled in which the Li foil and graphite electrodes were not electrically connected, although geometrically identical to the hybrid anode connected in series. When compared with traditional Li-S cells (Supplementary Fig. S1), there is a slight improvement in the cycling stability (Supplementary Fig. S5), indicating that the graphite does form a physical barrier that retards polysulphide transport, but the effect is minimal. Once connected with Li metal, the lithiated graphite goes beyond that of a simple diffusion barrier as discussed earlier. This hybrid anode design also differentiates from prelithiated graphite anode. Data collected on a simple $\mathrm{LiC}_{6} / \mathrm{S}$ full cell, using the same sulphur cathode but with a prelithiated graphite anode (without any Li foil connected), show poor electrochemical performance (Supplementary Fig. S6), further indicating that the synergistic effect of the connected $\mathrm{LiC}_{6}$ and $\mathrm{Li}$ metal anode is essential for performance enhancement ${ }^{49}$.

When graphite is replaced by hard carbon (Supplementary Fig. S7), the performance of the $\mathrm{Li}-\mathrm{S}$ cell is clearly inferior to that obtained from the cell using original $\mathrm{a} \mathrm{LiC}_{6} / \mathrm{Li}$ hybrid design. The lithiation/delithiation of hard carbon occurs over a much wide voltage range. This result suggests that it is likely to be that the hybrid anode may require an intercalation compound with an operating voltage close to that of Li metal to timely respond to the $\mathrm{Li}^{+}$extraction from the hybrid anode during charge. In addition, side reactions caused by the interactions between polysulphides and highly reductive $\mathrm{Li}$ occur throughout the whole electrochemical processes, as soluble polysulphides are generated in the beginning of the discharge and at the end of charge, which inevitably get involved in the SEI formation on the anode. The largely increased surface area of hard carbon nanowires (surface area: $75.8 \mathrm{~m}^{2} \mathrm{~g}^{-1}$ ) compared with that of graphite (surface area: $6.4 \mathrm{~m}^{2} \mathrm{~g}^{-1}$ ) provides more reactive sites for the parasitic reactions to initiate, weakening the hybrid anode effect, as can be reflected by the decreased Coulombic efficiency (Supplementary Fig. S7). Of note, the reduced performance of the hard carbon-containing anode is still a large improvement over a traditional Li-S cell construction (Supplementary Fig. S1).

From the above discussion, the function of the hybrid design is beyond that of a simple physical barrier. It involves an artificial and stable surface with a lithiation/delithiation voltage close to that of the Li metal and the needs to maintain the pseudo-equal potential through the shorted circuit. Findings to date suggest the lithiated graphite in the hybrid system functions similar to an 'artificial SEI layer'. Lithiated graphite negatives operate at a voltage only $\sim 150 \mathrm{mV}$ higher than that of Li metal. Theoretically, 
during discharge of $\mathrm{Li}-\mathrm{S}$ cells, $\mathrm{Li}^{+}$ions should first be extracted from Li metal instead of lithiated graphite $\left(\mathrm{LiC}_{6}\right)$. When shorted to Li metal, the lithiated graphite and the Li anode should be at equal potential. The charge/discharge rates used in this work are substantially higher than those used in conventional LIBs. $\mathrm{Li}^{+}$ ions will be released from both $\mathrm{LiC}_{6}$ and $\mathrm{Li}$ metal simultaneously, because the surface concentration of $\mathrm{Li}^{+}$ions around either surface is very low (close to zero) at such high current densities. Under this condition, the slight voltage difference is negligible and $\mathrm{Li}^{+}$ion contributions to the electrolyte may be largely from lithiated graphite-considering its physical location in the cell configuration. As graphite is always connected to Li metal, once $\mathrm{Li}^{+}$is removed from graphite the $\mathrm{Li}^{+}$cations will be automatically 'refilled' from the Li metal sink to eliminate the potential difference. In other words, lithiated graphite may function as a dynamic 'pump' that concurrently ejects $\mathrm{Li}^{+}$ions on demand to the working electrode and drains $\mathrm{Li}^{+}$ions from the Li metal reservoir to reload the graphite layers concurrently.

Now that the function of lithiated graphite during discharge has been discussed, what happens during charge needs to be considered. One major concern during charge is whether the $\mathrm{Li}^{+}$ ions will deposit on Li metal or lithiated graphite. At high charge rates, overlithiation, or Li plating, on the graphite has been reported $^{53}$. In the hybrid anode design, it is expected that $\mathrm{Li}+$ ions plate on both graphite and $\mathrm{Li}$ surface during charge. However, the relative amount of deposited $\mathrm{Li}$ on graphite and $\mathrm{Li}$ probably depends on the current density and the cell chemistry. In the presence of dissolved polysulphides, the composition of SEI on graphite is more complicated than in traditional cells, as it contains insulating sulphur species (Supplementary Fig. S3). The surface conductivity of lithiated graphite is lower than that of $\mathrm{Li}$ metal due to the existence of more insulating S-containing species on graphite film in the hybrid configuration. This is supported by the higher overpotential of $\mathrm{Li}$ deposition on lithiated graphite than on Li metal (Supplementary Fig. S8). Therefore, the overplating of $\mathrm{Li}$ on graphite surface is largely reduced.

In addition, multiple studies have shown that sulphurcontaining electrolytes help eliminate dendritic spikes on the graphite surface by chemically etching the protuberant $\mathrm{Li}^{46,53,54}$. Therefore, in the hybrid design, the graphite functions as a 'selfregulating' shuttle, which significantly reduces unwanted side reactions on the Li metal surface during both discharge and charge. It has to be pointed out that the reduced side reactions on Li side mainly refers to the interactions between polysulphides and highly reductive $\mathrm{Li}$, which continuously 'consume' active sulphur by forming a series of sulphur-containing byproducts (Supplementary Figs S2 and S3). The continuous consumption of active sulphur and formation of S-containing byproducts happen only when the microstructure of Li metal continues to grow. If there is no microstructure evolution such as porous structure formed from dendritic Li during cycling, the accumulation of those S-based byproducts will be largely limited to the surface instead of deeply penetrating into the interior of the Li metal. Therefore, the structure evolution of Li metal anode will help to understand how the hybrid anode works to prevent side reactions. With this in mind, we studied the structure evolution of Li metal anode using symmetric cells. Supplementary Fig. S9 shows that protected Li anode (hybrid Li anode) had much more dense structure (without dendrite) on the surface after cycling than that of unprotected one (with dendrite) in the symmetric cells, and that electrolyte decomposition still occurs on Li metal but is largely alleviated in the presence of the graphite film. In this hybrid anode design, the shuttle reaction still exists on graphite anode; thus, $\mathrm{LiNO}_{3}$ additive is still used in the electrolyte. However, the undesired reactions will not continuously grow, as the volume expansion of graphite is limited.
On the basis of these discussions, there are at least two basic requirements that have to be satisfied for the hybrid anode design. First, the electrochemically formed artificial SEI takes advantage of intercalation chemistry. Intercalation compound such as graphite undergoes minimum volume expansion (up to $10 \%$ ) during lithiation ${ }^{55}$. On repeated cycling, there is no structural damage on the graphite electrode that may expose new surfaces as parasite reaction sites. Second, minimum difference in operating voltages between the artificial SEI (lithiated graphite in this case) and $\mathrm{Li}$ metal ensures the timely refill of $\mathrm{Li}^{+}$ions into graphite lattice during the discharge process of $\mathrm{Li}-\mathrm{S}$ batteries (extraction of $\mathrm{Li}^{+}$from anode).

In summary, a radically different hybrid design has been introduced for Li-S cells to mitigate the loss of active material and harmful parasitic reactions on the anode by using an integrated structure composed of electrically connected graphite and $\mathrm{Li}$ metal. Although further studies are required, preliminary findings, combined with available literature results, support the idea that lithiated graphite functions as an electrochemical artificial SEI that decouples the $\mathrm{Li}^{+}$extraction/reinsertion from side reactions caused by soluble polysulphides. The anode reaction front is shifted from the Li metal to the graphite surface and the interaction with dissolved polysulphide species is primarily confined to graphite. The reduced irreversible sulphur loss on the Li metal anode, as well as the physical barrier benefit provided by the lithiated graphite, collectively leads to the exceptional electrochemical performances observed in $\mathrm{Li}-\mathrm{S}$ batteries. This simple hybrid concept provides important clues in the future development of Li-S batteries and may be broadly adapted in many energy-storage technologies utilizing metal anodes.

\section{Methods}

Cathode. A mesoporous carbon-sulphur (MCS) nanocomposite was used for both hybrid Li-S cell and traditional Li-S cell cathodes. The MCS composite contains an mesoporous carbon with a large pore volume $\left(22 \mathrm{~nm}, 4.8 \mathrm{~cm}^{3} \mathrm{~g}^{-1}\right)$ and controllable sulphur loading $(50 \mathrm{wt} \%)^{20}$. The cathode was prepared by mixing $80 \mathrm{wt} \%$ MCS nanocomposite powder, $10 \mathrm{wt} \%$ Super-P Li conductive carbon black (TIMCAL, Graphite \& Carbon Ltd) and $10 \mathrm{wt} \%$ polyvinylidene fluoride (Kynar HSV900, Arkema Inc.) binder in $N$-methylpyrrolidone onto aluminum foils and then dried at $55^{\circ} \mathrm{C}$ for $12 \mathrm{~h}$.

Hybrid anode. TIMREX SLP30 graphite powder (TIMCAL, Graphite \& Carbon Ltd) was mixed with Super-P (Timcal) and polyvinylidene fluoride in a ratio of 8:1:1 in $N$-methylpyrrolidone. The slurry was then coated onto porous stainless steel mesh and then connected with the Li metal foil. A piece of Celgard 2400 was placed in between the graphite film and $\mathrm{Li}$ metal to form the hybrid anode for $\mathrm{Li}-\mathrm{S}$ cells. It is critical that bonding fine metal filaments with carbon forms an interlocking composite network. The porous carbon-metallic grid composite electrode without carbon delamination or pinholes provides improved continuity of electrical conductivity and, hence, diminished Equivalent Series Resistance and advantageous modification of the macro-equivalent circuit.

Cell assembly. All of the coin cells (Type 2325 coin cell kits from CNRC, Canada) were assembled in an argon-filled glove box (MBraun, Inc.) with moisture and oxygen content of $<1$ p.p.m. Li metal disks were used as the anode. The electrolyte was $1 \mathrm{M}$ bis(trifluoromethane) sulphonimide Li salt (99.95\% trace metals basis, Sigma-Aldrich) dissolved in a mixture of 1,3-dioxolane (Sigma-Aldrich) and dimethoxymethane (Sigma-Aldrich; 1: 1 by volume). The dried and argon-protected $\mathrm{LiNO}_{3}$ (Sigma-Aldrich) additive was then added into the electrolyte to reach a concentration of $0.1 \mathrm{M}$.

Electrochemical tests. Electrochemical tests were performed using 2,325 coin cells (NRC, Canada) in ambient environment. The galvanostatic discharge-charge test was conducted using a BT-2043 Arbin Battery Testing System. All the cells were cycled at different current rates in the voltage interval of 1.0-3.0 V. All capacity values were calculated on the basis of sulphur mass. After cycling, several of the coin cells were disassembled in the charged or discharged state for further analysis.

Electrode characterization. To analyse the electrode properties and cell operation mechanisms, several of the coin cells were disassembled in the fresh, discharged or 
charged state, respectively. Each electrode, including Li anode, carbon anode and MCS cathode, was thoroughly washed in the glove box by a mixture solvent of 1,3dioxolane and dimethoxymethane (1:1 by volume) to remove any electrolyte salt residuals, and then transferred into vacuum chamber via a Sample Saver storage container (South Bay Technology, Inc.). Scanning electron microscopy experiments for the investigation of the electrode surface morphology and composition were performed on an FEI Quanta 3D FEG focused ion beam/scanning electron microscope equipped with an Oxford INCA PentFEXx3 system with $33 \mathrm{~mm}$ window EDAX detector. For quantitative comparison of S-elemental contents of $\mathrm{Li}$ anode surfaces from the half cell and full cell, S-elemental mapping with fixed frame collection counts (Res: $256 \times 200$ and Frame Reads: 500/500) was used to calculate atom\% of S K versus atom\% of C K. X-ray photoelectron spectroscopy measurements were performed with a Physical Electronics Quantera Scanning $\mathrm{X}$-ray Microprobe. This system uses a focused monochromatic $\mathrm{Al} K_{\alpha} \mathrm{X}$-ray $(1,486.7 \mathrm{eV})$ source for excitation and a spherical section analyser. The instrument has a 32-element multichannel detection system. A 100-W X-ray beam focused to $100 \mu \mathrm{m}$ diameter was rastered over a $1.4 \times 0.1 \mathrm{~mm}$ rectangle on the sample. The $\mathrm{X}$-ray beam is incident normal to the sample and the photoelectron detector is at $45^{\circ}$ off-normal. High-energy resolution (narrow scan) X-ray photoemission spectra were collected using a pass energy of $69.0 \mathrm{eV}$ with a step size of $0.125 \mathrm{eV}$. For the $\mathrm{Ag} 3 \mathrm{~d} 5 / 2$ line, these conditions produced a full width at half maximum of $0.91 \mathrm{eV}$. The sample experienced variable degrees of charge. All the spectra were charge referenced using $\mathrm{C} 1 \mathrm{~s}$ line at $285.0 \mathrm{eV}$ for comparison purpose. Low-energy electrons at $\sim 1 \mathrm{eV}, 20 \mu \mathrm{A}$ and low-energy $\mathrm{Ar}^{+}$ions were used to minimize this charge.

\section{References}

1. Bruce, P. G., Scrosati, B. \& Tarascon, J.-M. Nanomaterials for rechargeable lithium batteries. Angew. Chem. Int. Ed. 47, 2930-2946 (2008).

2. Dunn, B., Kamath, H. \& Tarascon, J.-M. Electrical energy storage for the grid: a battery of choices. Science 334, 928-935 (2011).

3. Yang, Z. et al. Electrochemical energy storage for green grid. Chem. Rev. 111, 3577-3613 (2011).

4. Kang, B. \& Ceder, G. Battery materials for ultrafast charging and discharging. Nature 458, 190-193 (2009).

5. Goodenough, J. B. \& Kim, Y. Challenges for rechargeable Li batteries. Chem. Mater. 22, 587-603 (2009).

6. Lee, S. W. et al. High-power lithium batteries from functionalized carbonnanotube electrodes. Nat. Nanotech. 5, 531-537 (2010).

7. Gao, X.-P. \& Yang, H.-X. Multi-electron reaction materials for high energy density batteries. Energy Environ. Sci. 3, 174-189 (2010).

8. Whittingham, M. S. Lithium batteries and cathode materials. Chem. Rev. 104, 4271-4302 (2004).

9. Tarascon, J. M. \& Armand, M. Issues and challenges facing rechargeable lithium batteries. Nature 414, 359-367 (2001).

10. Bruce, P. G., Freunberger, S. A., Hardwick, L. J. \& Tarascon, J.-M. Li- $\mathrm{O}_{2}$ and Li-S batteries with high energy storage. Nat. Mater. 11, 19-29 (2012).

11. Su, Y. \& Manthiram, A. Lithium-sulphur batteries with a microporous carbon paper as a bifunctional interlayer. Nat. Commun. 3, 1166 (2012).

12. Ji, X. \& Nazar, L. F. Advances in Li-S batteries. J. Mater. Chem. 20, $9821-9826$ (2010).

13. Aurbach, D. et al. On the surface chemical aspects of very high energy density, rechargeable Li-sulfur batteries. J. Electrochem. Soc. 156, A694-A702 (2009).

14. Peramunage, D. \& Licht, S. A solid sulfur cathode for aqueous batteries. Science 261, 1029-1032 (1993).

15. Mikhaylik, Y. V. \& Akridge, J. R. Polysulfide shuttle study in the Li/S battery system. J. Electrochem. Soc. 151, A1969-A1976 (2004).

16. Ji, X., Lee, K. T. \& Nazar, L. F. A highly ordered nanostructured carbon-sulphur cathode for lithium-sulphur batteries. Nat. Mater. 8, 500-506 (2009).

17. Hassoun, J. \& Scrosati, B. A high-performance polymer tin sulfur lithium ion battery. Angew. Chem. Int. Ed. 49, 2371-2374 (2010).

18. Hassoun, J. \& Scrosati, B. Moving to a solid-state configuration: a valid approach to making lithium-sulfur batteries viable for practical applications. Adv. Mater. 22, 5198-5201 (2010).

19. Ji, L. et al. Graphene oxide as a sulfur immobilizer in high performance lithium/ sulfur cells. J. Am. Chem. Soc. 133, 18522-18525 (2011).

20. Li, X. et al. Optimization of mesoporous carbon structures for lithium-sulfur battery applications. J. Mater. Chem. 21, 16603-16610 (2011).

21. Ji, X., Evers, S., Black, R. \& Nazar, L. F. Stabilizing lithium-sulphur cathodes using polysulphide reservoirs. Nat. Commun. 2, 325 (2011).

22. Jayaprakash, N., Shen, J., Moganty, S. S., Corona, A. \& Archer, L. A. Porous hollow carbon@sulfur composites for high-power lithium-sulfur batteries. Angew. Chem. Int. Ed. 50, 5904-5908 (2011).

23. Elazari, R., Salitra, G., Garsuch, A., Panchenko, A. \& Aurbach, D. Sulfurimpregnated activated carbon fiber cloth as a binder-free cathode for rechargeable Li-S batteries. Adv. Mater. 23, 5641-5644 (2011).

24. Ji, L. et al. Porous carbon nanofiber-sulfur composite electrodes for lithium/ sulfur cells. Energy Environ. Sci. 4, 5053-5059 (2011).
25. Liang, C., Dudney, N. J. \& Howe, J. Y. Hierarchically structured sulfur/carbon nanocomposite material for high-energy lithium battery. Chem. Mater. 21, 4724-4730 (2009).

26. Schuster, J. et al. Spherical ordered mesoporous carbon nanoparticles with high porosity for lithium-sulfur batteries. Angew. Chem. Int. Ed. 51, 3591-3595 (2012).

27. Demir-Cakan, R. et al. Cathode composites for Li-S batteries via the use of oxygenated porous architectures. J. Am. Chem. Soc. 133, 16154-16160 (2011).

28. Cao, Y. et al. Sandwich-type functionalized graphene sheet-sulfur nanocomposite for rechargeable lithium batteries. Phys. Chem. \& Chem. Phys. 13, 7660-7665 (2011).

29. Yang, Y. et al. New nanostructured $\mathrm{Li}_{2} \mathrm{~S} /$ silicon rechargeable battery with high specific energy. Nano Lett. 10, 1486-1491 (2010).

30. Wu, F. et al. Sulfur/polythiophene with a core/shell structure: Synthesis and electrochemical properties of the cathode for rechargeable lithium batteries. J. Phys. Chem. C 115, 6057-6063 (2011).

31. Wang, J. et al. Sulfur composite cathode materials for rechargeable lithium batteries. Adv. Funct. Mater. 13, 487-492 (2003).

32. Peled, E., Gorenshtein, A., Segal, M. \& Sternberg, Y. Rechargeable lithiumsulfur battery (extended abstract). J. Power Sources 26, 269-271 (1989).

33. Suo, L., Hu, Y.-S., Li, H., Armand, M. \& Chen, L. A new class of solvent-in-salt electrolyte for high-energy rechargeable metallic lithium batteries. Nat. Commun. 4, 1481 (2013).

34. Affinito, J. In Symp. Beyond Li-ion Batteries (Oak Ridge National Laboratory, 2010).

35. Liang, C. Carbon/sulfur Nanocomposites and Additives for High-Energy Lithium Sulfur Batteries. Vehicle Technologies Program Annual Merit Review and Peer Evaluation Meeting, Washington DC (2011).

36. Shin, J. H. \& Cairns, E. J. Characterization of N-methyl-N-butylpyrrolidinium bis(trifluoromethanesulfonyl)imide-LiTFSI-tetra(ethylene glycol) dimethyl ether mixtures as a Li metal cell electrolyte. J. Electrochem. Soc. 155, A368-A373 (2008).

37. Zhang, S. S. \& Read, J. A. A new direction for the performance improvement of rechargeable lithium/sulfur batteries. J. Power Sources 200, 77-82 (2012).

38. Barchasz, C., Leprêtre, J.-C., Alloin, F. \& Patoux, S. New insights into the limiting parameters of the $\mathrm{Li} / \mathrm{S}$ rechargeable cell. J. Power Sources 199, 322-330 (2012).

39. Xiao, L. et al. A soft approach to encapsulate sulfur: Polyaniline nanotubes for lithium-sulfur batteries with long cycle life. Adv. Mater. 24, 1176-1181 (2012).

40. Hayashi, A., Ohtomo, T., Mizuno, F., Tadanaga, K. \& Tatsumisago, M. Allsolid-state $\mathrm{Li} / \mathrm{S}$ batteries with highly conductive glass-ceramic electrolytes. Electrochem. Commun. 5, 701-705 (2003).

41. Hassoun, J., Sun, Y.-K. \& Scrosati, B. Rechargeable lithium sulfide electrode for a polymer tin/sulfur lithium-ion battery. J. Power Sources 196, 343-348 (2011)

42. Hassoun, J. et al. A contribution to the progress of high energy batteries: a metal-free, lithium-ion, silicon-sulfur battery. J. Power Sources 202, 308-313 (2012).

43. Wang, J., Yao, Z., Monroe, C. W., Yang, J. \& Nuli, Y. Carbonyl-betacyclodextrin as a novel binder for sulfur composite cathodes in rechargeable lithium batteries. Adv. Funct. Mater. 23, 1194-1201 (2013).

44. Aurbach, D., Zinigrad, E., Teller, H. \& Dan., P. Factors which limit the cycle life of rechargeable lithium (metal) batteries. J. Electrochem. Soc. 147, 1274-1279 (2000).

45. Vaughey, J. \& Carmen Lopez, D. D. Lithium Metal Anodes. Vehicle Technologies Program Annual Merit Review and Peer Evaluation Meeting, Washington DC (2009).

46. Mikhaylik, Y. et al. High energy rechargeable Li-S cells for EV application. Status, remaining problems and solutions. ECS Transactions 25, 23-34 (2010).

47. Zheng, J. et al. Ionic liquid-enhanced solid state electrolyte interface (SEI) for lithium-sulfur batteries. J. Mater. Chem. A 1, 8464-8470 (2013).

48. Demir-Cakan, R. et al. Li-S batteries: simple approaches for superior performance. Energy Environ. Sci. 6, 176-182 (2013).

49. Schneider, H. et al. Influence of different electrode compositions and binder materials on the performance of lithium-sulfur batteries. J. Power Sources 205, 420-425 (2012)

50. Diao, Y., Xie, K., Xiong, S. \& Hong, X. Analysis of polysulfide dissolved in electrolyte in discharge-charge process of Li-S battery. J. Electrochem. Soc. 159, A421-A425 (2012)

51. Chusid, O., Ein-Ely, Y. E. \& Aurbach, D. Electrochemical and spectroscopic studies of carbon electrodes in lithium battery electrolyte system. J. Power Sources 43-44, 47-64 (1993).

52. Aurbach, D., Ein-Eli, Y. \& Chusid, O. The correlation between the surface chemistry and the performance of Li-carbon intercalation anodes for reachargeable 'rocking-chair'type batteries. J. Electrochem. Soc. 141, 603-611 (1994).

53. Lu, W. et al. Overcharge effect on morphology and structure of carbon electrodes for lithium-ion batteries. J. Electrochem. Soc. 159, A566-A570 (2012). 
54. Wagner, M. W., Liebenow, C. \& Besenhard, J. O. Effect of polysulfidecontaining electrolyte on the film formation of the negative electrode. J. Power Sources 68, 328-332 (1997).

55. Reynier, Y., Yazami, R. \& Fultz, B. XRD evidence of macroscopic composition inhomogeneities in the graphite-lithium electrode. J. Power Sources 165, 616-619 (2007).

\section{Acknowledgements}

The development and demonstration of the hybrid design are supported by the U.S Department of Energy (DOE), Office of Basic Energy Sciences, Division of Materials Sciences and Engineering, under Award KC020105-FWP12152. The mechanistic study on the symmetric cell and over potentials of the electrode materials were supported by DOE's Advanced Research Projects Agency-Energy. The cathode used in this work is supported by the Assistant Secretary for Energy Efficiency and Renewable Energy, Office of Vehicle Technologies of the U.S. Department of Energy under contract number DE-AC02-05CH11231 and subcontract number 18769 under the Batteries for Advanced Transportation Technologies (BATT) programme. The scanning electron microscopy, transmission electron microscopy, energy dispersive spectroscopy and X-ray photoelectron spectroscopy were conducted at the Environmental and Molecular Sciences Laboratory, a national scientific user facility sponsored by the Department of Energy's Office of Biological and Environmental Research and located at Pacific Northwest
National Laboratory (PNNL). PNNL is a multiprogramme national laboratory operated for DOE by Battelle under contract DE AC05-76RL01830.

\section{Author contributions}

J.L. and C.H. conceived and designed this work; J.X. and J.L. proposed the mechanism and wrote the manuscript; C.H., Y.S., J. Zhe., W.D.B. and D.L. performed the experiment; S.V.L. and M.E. did the characterization; L.J. and G.L.G. participated in the manuscript editing, X.L. provided the mesoporous carbon; J. Zha. and G.L.G. participated in the discussion of this work.

\section{Additional information}

Supplementary Information accompanies this paper at http://www.nature.com/ naturecommunications

Competing financial interests: The authors declare no competing financial interests.

Reprints and permission information is available online at http://npg.nature.com/ reprintsandpermissions/

How to cite this article: Huang, C. et al. Manipulating surface reactions in lithium-sulphur batteries using hybrid anode structures. Nat. Commun. 5:3015 doi: 10.1038/ncomms4015 (2014). 


\section{Corrigendum: Manipulating surface reactions in lithium-sulphur batteries using hybrid anode structures}

Cheng Huang, Jie Xiao, Yuyan Shao, Jianming Zheng, Wendy D. Bennett, Dongping Lu, Laxmikant V. Saraf, Mark Engelhard, Liwen Ji, Jiguang Zhang, Xiaolin Li, Gordon L. Graff \& Jun Liu

Nature Communications 5:3015 doi: 10.1038/ncomms4015 (2014); Published 9 Jan 2014; Updated 20 Feb 2014

The original version of this Article contained an error in the order in which the first and last name of the author Laxmikant V. Saraf were displayed. This has now been corrected in both the PDF and HTML versions of the Article. 